\title{
The capsule of Porphyromonas gingivalis reduces the immune response of human gingival fibroblasts
}

\author{
Jorg Brunner ${ }^{1}$, Nina Scheres ${ }^{1}$, Nawal B El Idrissi', Dong M Deng ${ }^{1}$, Marja L Laine ${ }^{1}$, Arie J van Winkelhoff², \\ Wim Crielaard $^{1 *}$
}

\begin{abstract}
Background: Periodontitis is a bacterial infection of the periodontal tissues. The Gram-negative anaerobic bacterium Porphyromonas gingivalis is considered a major causative agent. One of the virulence factors of $P$. gingivalis is capsular polysaccharide (CPS). Non-encapsulated strains have been shown to be less virulent in mouse models than encapsulated strains.

Results: To examine the role of the CPS in host-pathogen interactions we constructed an insertional isogenic $P$. gingivalis knockout in the epimerase-coding gene epsC that is located at the end of the CPS biosynthesis locus. This mutant was subsequently shown to be non-encapsulated. K1 capsule biosynthesis could be restored by in trans expression of an intact epsC gene. We used the epsC mutant, the W83 wild type strain and the complemented mutant to challenge human gingival fibroblasts to examine the immune response by quantification of $I L-1 \beta, I L-6$ and $I L-8$ transcription levels. For each of the cytokines significantly higher expression levels were found when fibroblasts were challenged with the epsC mutant compared to those challenged with the W83 wild type, ranging from two times higher for $\mathrm{IL}-1 \beta$ to five times higher for IL-8.
\end{abstract}

Conclusions: These experiments provide the first evidence that $P$. gingivalis CPS acts as an interface between the pathogen and the host that may reduce the host's pro-inflammatory immune response. The higher virulence of encapsulated strains may be caused by this phenomenon which enables the bacteria to evade the immune system.

\section{Background}

Porphyromonas gingivalis is a major pathogen in destructive periodontal diseases including chronic and aggressive periodontitis that are characterized by breakdown of the tooth-supporting tissues [1-3]. P. gingivalis is a black pigmented, often encapsulated, strict anaerobic, Gram negative coccobacillus that occurs in the human oral cavity.

Among the variety of virulence factors that have been described for $P$. gingivalis, CPS has shown to be a major factor in experimental infections. Studies in a mouse infection model have revealed that encapsulated $P$. gingivalis strains are more virulent than non-encapsulated

\footnotetext{
* Correspondence: w.crielaard@acta.nl

'Department of Oral Microbiology, Academic Centre for Dentistry

Amsterdam, Universiteit van Amsterdam and Vrije Universiteit, Amsterdam,
} The Netherlands strains [4-7]. Non-encapsulated strains mostly cause non-invasive, localized abscesses whereas encapsulated strains cause invasive, spreading phlegmonous infections after subcutaneous inoculation of experimental animals.

Six distinct capsular serotypes have currently been described (K1-K6) [8,9] and a seventh serotype (K7) has been suggested by R. E. Schifferle (personal communication). Small differences in virulence have been found between capsular serotypes and strong variation in virulence has been described between strains of the same capsular serotype [10]. CPS of all serotypes has been tested for induction of immunological responses in macrophages and it has been revealed that the CPS of K1 serotype strains induces higher chemokine expression in murine peritoneal macrophages than the other serotypes [11]. These data suggest that the K1 CPS plays an important role in host-pathogen interaction. The 
chemical composition of the K1 CPS has been studied to a limited extent. It has been reported that the CPS of K1 (strain W50) comprises of mannuronic acid (ManA), glucuronic acid (GlcA), galacturonic acid (GalA), galactose and $\mathrm{N}$-acetylglucosamine (GlcNAc), but the CPS structure has not been solved [12].

Although CPS is a major structure at the interface between the bacterial cell and the host, the exact role of $P$. gingivalis CPS is not yet clear. Adhesion to epithelial cells has been shown to be higher for non-encapsulated $P$. gingivalis and the level and mechanism of co-aggregation has been shown to be CPS dependent [5,13,14]. In many pathogens CPS has been found to be involved in evasion of the host immune system by circumvention of phagocytosis, opsonization and complement killing [15-17].

The aim of this study was to investigate in vitro differences in host response during infection with a wild type and an isogenic non-encapsulated mutant of a naturally encapsulated strain. The well-studied K1 serotype W83 strain was used as the wild type strain since its CPS biosynthesis locus has been described $[18,19]$. An insertional mutation in PGO120 (epsC) was constructed, which yielded a non-encapsulated strain. The gene has been annotated as a UDP-GlcNAc 2-epimerase.

This eps $C$ mutant is tested in a fibroblast infection model [20] since fibroblasts are the most abundant stromal cells in soft connective tissue of the gingiva [21] and among the first cells encountering periodontal infections by anaerobic bacteria like $P$. gingivalis. And above all, fibroblasts have been shown to be involved in the immune response in periodontitis [22,23]. Human gingival fibroblasts were infected with W83 and the eps $C$ mutant and transcription of $I L-1 \beta, I L-6$ and $I L-8$ was determined as host response parameters. This study provides the first direct evidence that $P$. gingivalis CPS reduces the host immune response, thereby potentially enabling evasion of the immune system to sustain successful long-term infection.

\section{Results}

\section{EpsC mutant construction}

After transformation of the linearized plasmid $\mathrm{p} \Delta \mathrm{EpsC}$ to $P$. gingivalis W83 the epsC insertional mutation was confirmed by specific PCR amplifications and agarose gel electrophoresis of the products (data not shown). Primer combinations epsC BamHI F $\times$ PG0119 R and EryF $\mathrm{F} \times$ epsC EcoRI $\mathrm{R}$ (Table 1) ensured that a $1.2 \mathrm{~Kb}$ fragment of $\mathrm{p} \Delta \mathrm{EpsC}$ had been integrated by double crossover at PG0120 (epsC) as expected, replacing the intact copy with the insertionally inactivated copy (Figure 1).
Table 1 Primers used in this study

\begin{tabular}{|c|c|c|}
\hline Target & Name & Sequence $\left(5^{\prime}-3^{\prime}\right)$ \\
\hline \multirow[t]{4}{*}{ epsC } & $\begin{array}{l}\text { epsC } \\
\text { BamHI F }\end{array}$ & ATATAGGATCCATGAAAAAAGTGATGTTGGTC \\
\hline & $\begin{array}{l}\text { epsC EcoRl } \\
\mathrm{R}\end{array}$ & CTATGAATTCATCTTCGGCTAAATGCATCG \\
\hline & epsC Ascl F & GAATATAGGCGCGCCATGAAAAAAGTGATGTTGGTC \\
\hline & epsC Spel & CTATACTAGTATCTTCGGCTAAATGCATCG \\
\hline \multirow[t]{2}{*}{ eryF } & eryF Clal F & CCACCATCGATCGATAGCTTCCGCTATTGC \\
\hline & eryF Clal R & CCACCATCGATGTTTCCGCTCCATCGCCAATTTGC \\
\hline \multirow[t]{2}{*}{ CP25 } & CP25 Clal F & GCCATATCGATGCATGCGGATCCCATTATG \\
\hline & CP25 Ascl R & CCTITAGGCGCGCCCTTAATTTCTCCTC \\
\hline \multirow[t]{2}{*}{ IL-6 } & IL-6 F & GGCACTGGCAGAAAACAACC \\
\hline & IL-6 R & GGCAAGTCTCCTCATTGAATCC \\
\hline \multirow[t]{2}{*}{$\operatorname{lL}-8$} & IL-8 F & GGCAGCCTTCCTGATTTCTG \\
\hline & IL-8 R & CTGACACATCTAAGTTCTTCTTTAGCACTCCTT \\
\hline \multirow[t]{2}{*}{$\mathbb{L L}-1 \beta$} & $\mathrm{IL}-1 \beta \mathrm{F}$ & AAGATTCAGGTTTACTCACGTC \\
\hline & $\mathrm{IL}-1 \beta \mathrm{R}$ & TGATGCTGCTTACATGTCTCG \\
\hline \multirow[t]{2}{*}{ hup-1 } & hup-1 F & GAAAAGGCCAACCTCACAAA \\
\hline & hup-1 F & TCCGATGAGAGCGATTTCT \\
\hline \multirow[t]{2}{*}{$g / k$} & glk F & ATGAATCCGATCCGCCACCAC \\
\hline & glk R & GCCTCCCATCCCAAAGCACT \\
\hline
\end{tabular}

In bold: restriction sites used in this study

To examine if the mutation had an influence on the growth characteristics of the eps $C$ mutant both W83 and the epsC mutant were grown in brain heart infusion broth supplemented with hemin $(5 \mu \mathrm{g} / \mathrm{ml})$ and menadione $(1 \mu \mathrm{g} / \mathrm{ml})(\mathrm{BHI}+\mathrm{H} / \mathrm{M})$. Phase-contrast microscopy revealed that the mutant grows in aggregates, but no difference in growth rate was observed.

\section{EpsC mutant characterization}

The potential polar effect of the insertional inactivation on the down stream gene of epsC named hup-1 was examined. Total RNA was extracted from W83 and the eps $C$ mutant in the early exponential phase and the hup-1 expression levels were evaluated by Real-Time PCR. No significant difference in expression of hup-1 was found between W83 and the epsC mutant (data not shown).

To show the effect of capsule-loss on the surface structure of $P$. gingivalis the hydrophobicity of the epsC mutant was tested by the capacity to adhere to hexadecane. While $3 \%$ of W83 cells was shown to adhere to hexadecane more than $60 \%$ of the eps $C$ mutant cells was adhered to hexadecane. $19 \%$ of the complemented mutant cells was adhered to hexadecane (see Additional file 1).

Reactivity with the CPS-specific polyclonal rabbit antisera against $P$. gingivalis serotypes $\mathrm{K} 1-\mathrm{K} 6$ [8,9] was 


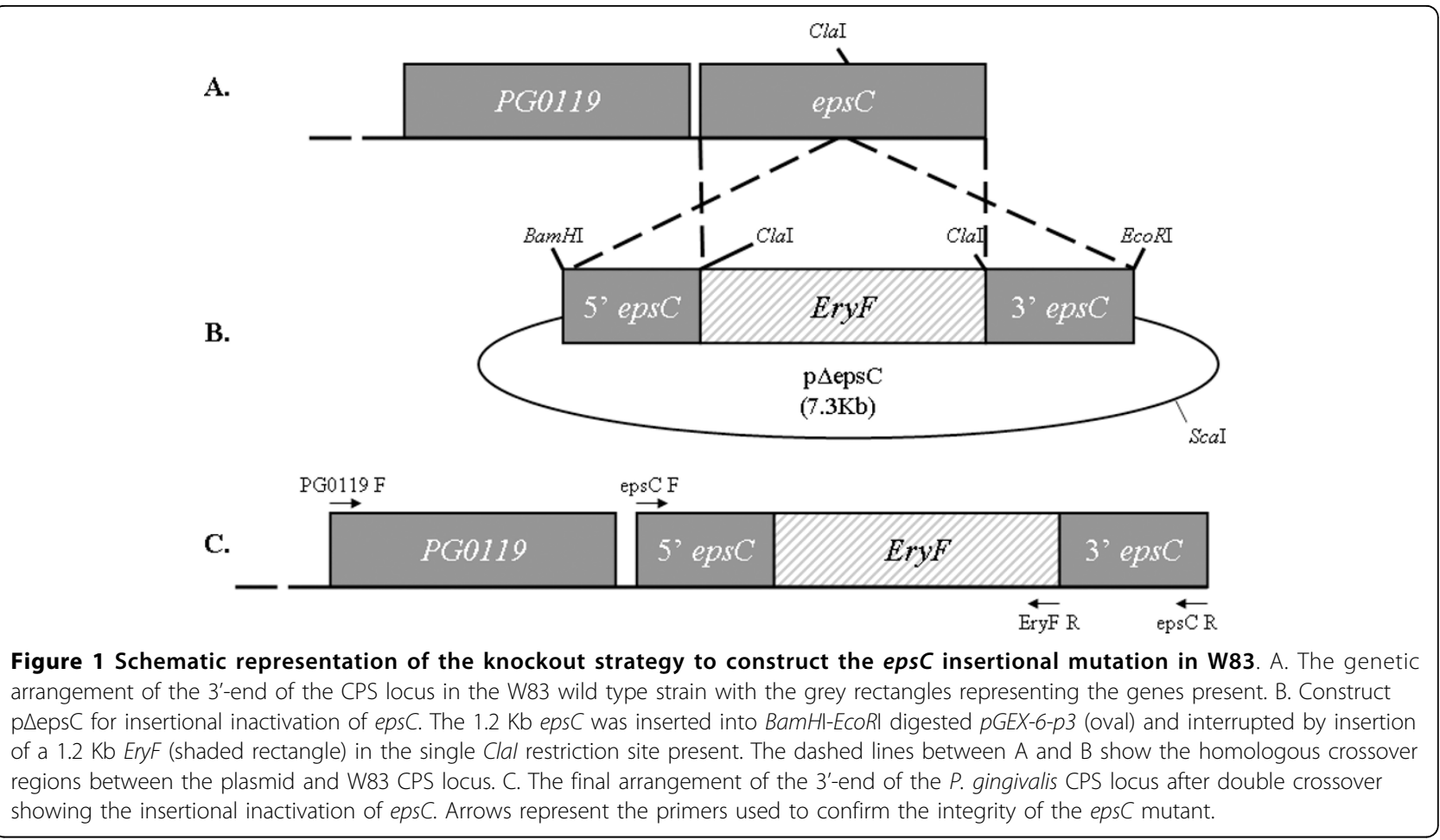

examined for W83 and the epsC mutant. The epsC mutant was not recognized by any of the antisera including the $\mathrm{K} 1$ antiserum, whereas the wild type strain was only recognized by the $\mathrm{K} 1$ antiserum (Figure 2). Differences in CPS characteristics were also studied by Percoll density gradient centrifugation, which can reveal density differences between encapsulated and nonencapsulted bacteroides strains [24]. Percoll density gradient centrifugation analyses of W83 and the epsC mutant showed that the density of the mutant had been changed (Figure 3). Where W83 mostly settled at the 20-30\% interface, the epsC mutant settled at the 50-60\% interface. Note that the appearance of W83 is diffuse and not restricted to the $20-30 \%$ interface. The mutant settles as a compact and granulous layer.

To conclusively examine the absence of CPS in the eps $C$ mutant, light microscopy was performed using India ink in combination with fuchsine staining (Figure 4). The negative India ink staining allows direct visualization of the capsule, appearing as a light halo surrounding the $P$. gingivalis cell. Fuchsine is used to stain the cell body. The halos around the W83 wild type strain are clearly visible in the phase contrast microscopic picture, whereas halos are absent around the eps $C$ mutant. The intact eps $C$ gene in trans under control of the CP25 promoter rescues the wild-type phenotype enabling the complemented mutant to produce a K1 capsule again (Figures 2 and 4).

\section{Fibroblast response to $P$. gingivalis challenge}

To study the effect of the epsC deletion on the host immune response six hour infection studies of human gingival fibroblasts with $\mathrm{W} 83$ and the epsC mutant were performed. Figure 5 shows $I L-1 \beta, I L-6$ and $I L-8$ expression of infected gingival fibroblasts relative to the noninfected negative control which is set to 1 and normalized against expression of housekeeping gene GAPDH.

At multiplicity of infection (MOI) 1000:1 of both strains a small induction of the tested genes could be detected compared to the non-infected control, but significant induction for all three genes was found when MOI 10.000:1 was used for infection.At MOI 1000:1 IL6 and $I L-8$ expression showed a significantly higher induction (150-fold and 37-fold induction respectively) in the cells challenged with the epsC mutant when compared to the wild-type (6-fold and 2-fold induction respectively), $I L-1 \beta$ did not show a difference compared to the wild-type. However, when gingival fibroblasts were challenged with MOI 10.000 bacteria all three tested genes showed a significantly higher induction in the cells challenged with the epsC mutant than with W83 (figure 5). When fibroblasts were challenged with the complemented mutant the response was almost completely restored to wild-type levels (see Additional file 2).

Sedimentation of the epsC mutant in comparison to W83 was analyzed in the same buffer as used in the infection experiments. No significant sedimentation 


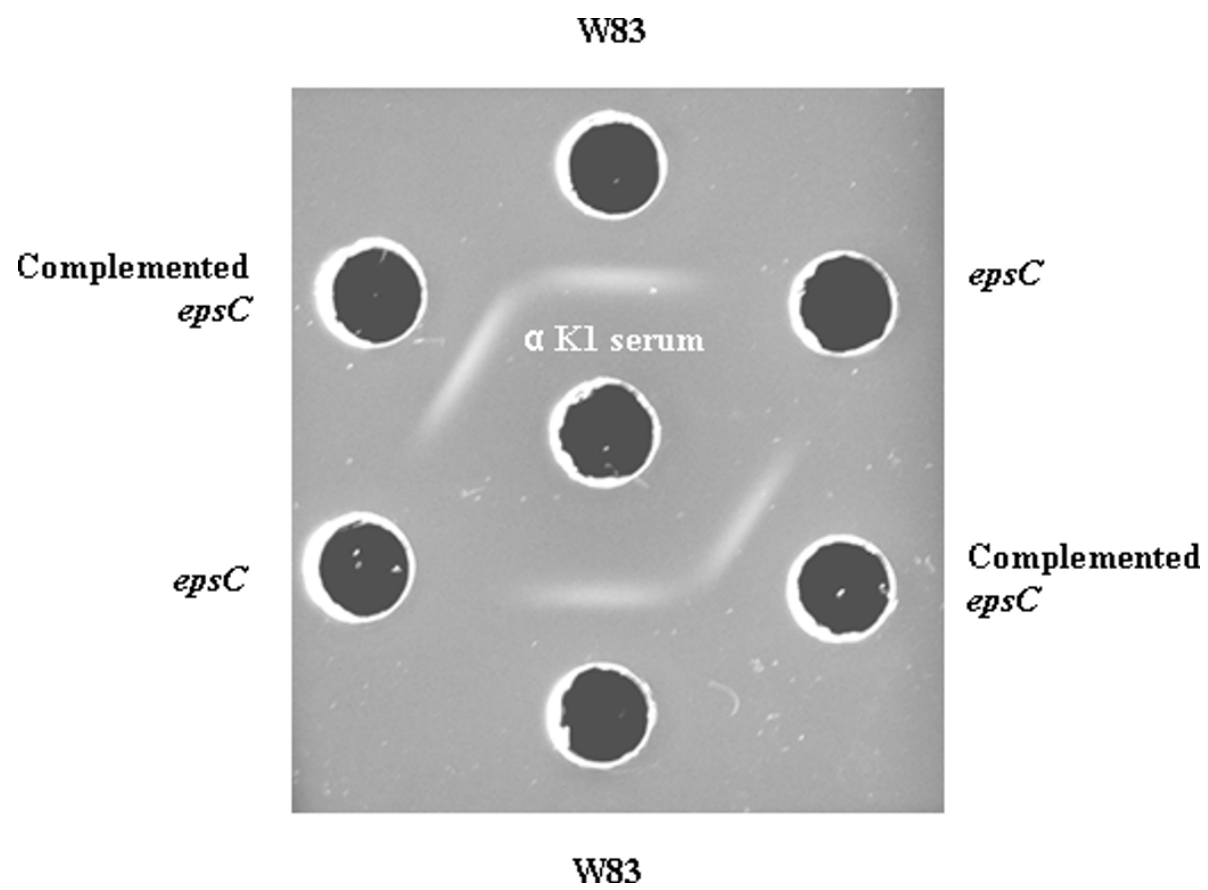

Figure 2 Double immunodiffusion analysis of autoclaved supernatants of $\boldsymbol{P}$. gingivalis strains. Samples of W83, the epsC mutant and the complemented mutant were tested against the K1-specific antiserum (central well). Note that the white precipitate indicating recognition of CPS with the antiserum is absent in case of the epsC mutant, whereas the intact epsC copy restores the wild-type $\mathrm{K} 1$ antiserum recognition in the complemented mutant.

differences were found between W83 and the epsC mutant within the 6 hours needed for infection of the fibroblasts (data not shown).

Since infections were done with viable $P$. gingivalis, survival of the bacteria during the 6-hour aerobic period of infection in DMEM medium had to be ensured. Therefore a 6-hour survival experiment was performed in the 24-well plates used for the fibroblast challenge. On average $60-75 \%$ of W83, epsC mutant and complemented mutant cells survived for 6 hours in Dulbecco's modified Eagle's Medium (DMEM; Sigma Chemical Co.) supplemented with $10 \%$ fetal calf serum (FCS) (see Additional file 3).

\section{Discussion}

The aim of this paper was to understand the role of $P$. gingivalis CPS in the response of human gingival fibroblasts.P. gingivalis CPS has been regarded as an important virulence factor. It has been shown to induce inflammatory mediators in in vitro studies [11]. The capsule also plays an important role in shielding of immune response inducers in several bacterial species [25-27]. Since a distinct CPS biosynthesis locus in $P$. gingivalis has been described and shown to be functional $[18,19]$, studying the role of $P$. gingivalis CPS in the immune response by use of a mutant became feasible. For this purpose an insertional isogenic knockout in
epsC, a potential capsular biosynthesis gene within the CPS biosynthesis locus present in strains of different serotypes, was constructed to prevent capsule synthesis. The homologue of this gene in Listeria monocytogenes lmo2537 has been shown to be essential for survival, and has been suggested to be involved in the maintenance of cell shape by providing a precursor of the teichoic acid linkage unit that serves as an acceptor for the main teichoic acid chain assembly [28]. Construction of the $P$. gingivalis eps $C$ mutant shows that the epsC gene is not essential for $P$. gingivalis viability.

In the present study the mutant is shown to be nonencapsulated by double immuno-diffusion, density gradient centrifugation and India ink staining. Complementation resulted in rescue of wild-type $\mathrm{K} 1$ capsule biosynthesis. Although the exact role of eps $C$ remains to be elucidated, this finding provides evidence that EpsC is essential in $P$. gingivalis CPS biosynthesis.

The epsC mutant was expected to have altered immunological properties. To examine the role of CPS, both the wild-type and the epsC mutant were used in an in vitro challenge of primary human gingival fibroblasts. Since the epsC mutant has altered physical properties, it was important to compare the sedimentation rate and viability of both the wild type and the mutant strain since these could have influenced the amount of living bacterial cells that are in contact with the fibroblasts. 


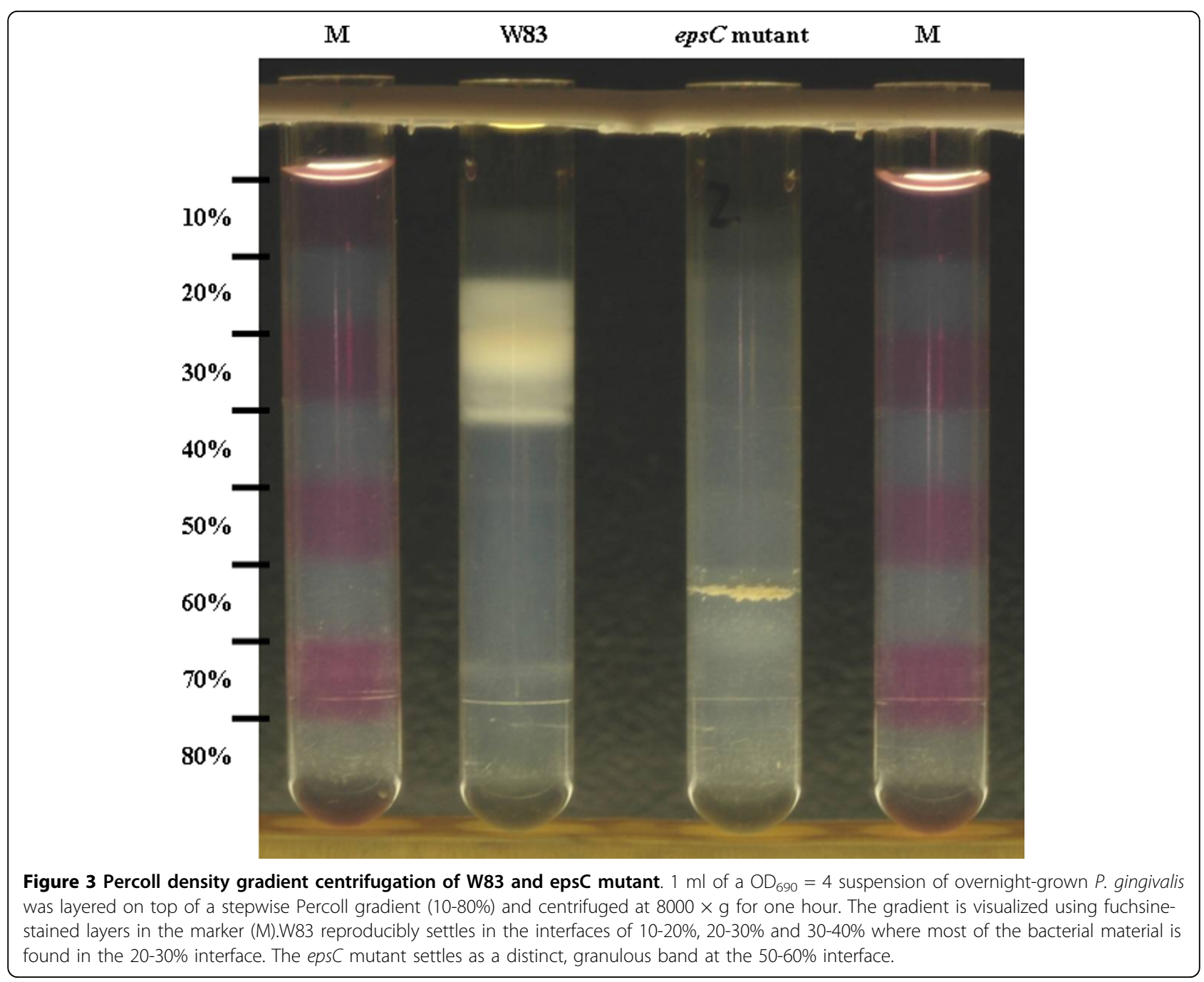

No differences were observed between the strains during the 6 hours of infection.

From the infection experiments of the gingival fibroblasts it became apparent that pro-inflammatory mediators $I L-1 \beta, I L-6$ and $I L-8$ expression levels were upregulated after a 6-hour challenge with both wild-type W83 and the epsC mutant in comparison to the noninfected control, especially when MOIs of 10.000:1 were used.

A challenge with the eps $C$ mutant induced a significantly higher pro-inflammatory immune response than a challenge with the wild type W83, as shown by $I L-1 \beta$, $I L-6$ and $I L-8$ gene expression. So, even though purified $P$. gingivalis CPS has been shown to stimulate proinflammatory cytokine expression in murine peritoneal macrophages [11] the absence of capsule induces extra cytokine induction when viable $P$. gingivalis cells were used to challenge fibroblasts.
Capsular polysaccharides of several bacteria have been implicated in down-regulation of pro-inflammatory cytokine production, including Klebsiella pneumonia [29]. Bacteroides fragilis capsular polysaccharide complex has been shown to induce IL-10 expression, a regulating cytokine which may cause suppression of the immune system [30].

An explanation of our results may be that the CPS prevents more potent immune inducers to be recognized by Toll-like receptors on the fibroblasts. It has been shown that the capsular antigen in Salmonella typhi, referred to as $\mathrm{Vi}$-antigen, is able to prevent Tolllike receptor 4 recognition of LPS, thereby reducing expression of pro-inflammatory TNF- $\alpha$ and IL- 6 [31-33]. In E. coli the capsule may cover short (10 nm) bacterial adhesins, which do not penetrate the 0.2-1.0 $\mu \mathrm{m}$ capsular layer, preventing them from being recognized by the immune system [26]. Likewise, $P$. gingivalis strain W83 was described as to have a small amount of 

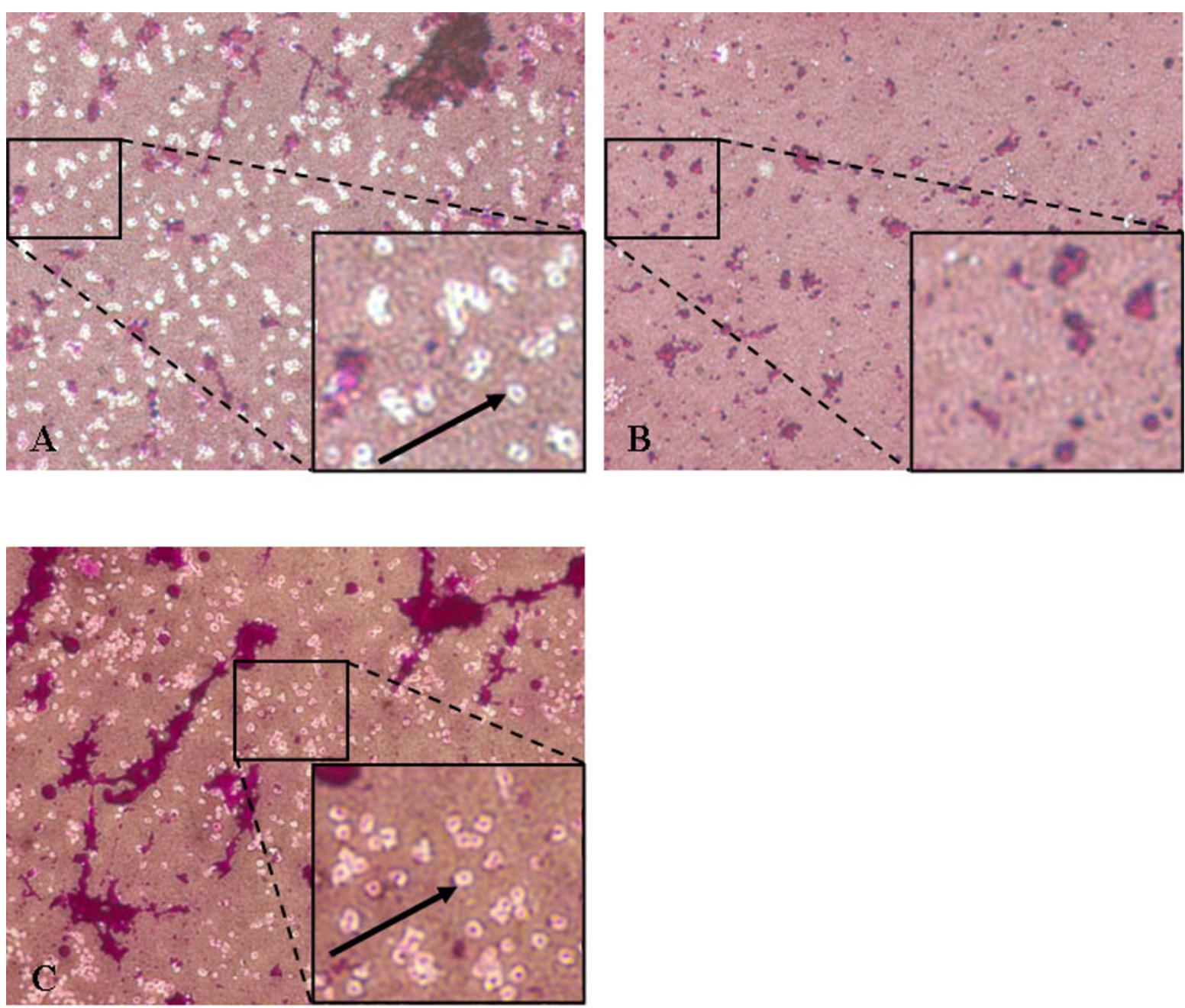

Figure 4 Negative capsule staining of fuchsine-stained P. gingivalis cells with India Ink. Phase contrast microscopic picture at a 1000x magnification of (A) W83 wild type strain, (B) epsC mutant and (C) the complemented epsC mutant in an India ink preparation which reveals the capsule as a white halo (arrow). The inset shows an extra six times magnification.

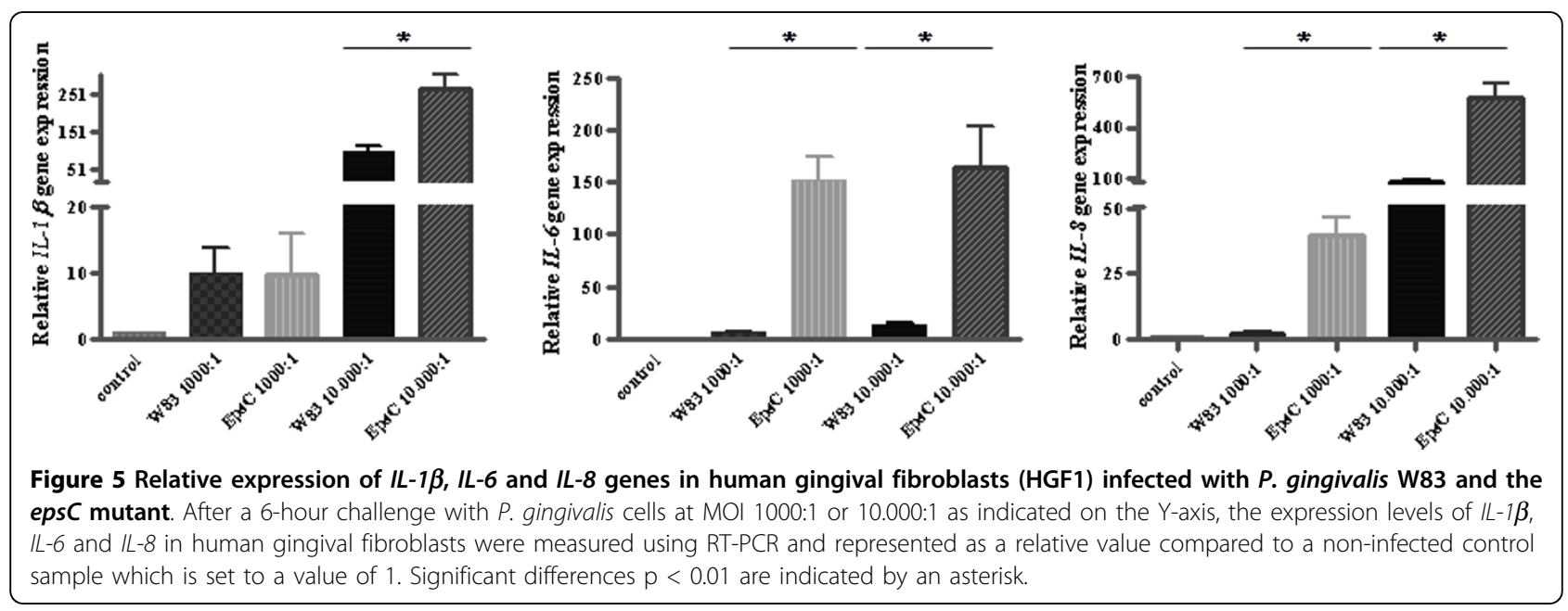


short fimbriae that might be mostly covered by the CPS [34].

Another or additional explanation of our findings could be immune suppression by $P$. gingivalis CPS, meaning that CPS would actively modulate the immune response of the fibroblasts, leading to lower inflammatory cytokine expression levels, potentially enabling $P$. gingivalis to evade the immune system.

For several bacteria it has been described that capsular biosynthesis can be modulated depending on environmental conditions $[35,36]$. Although presently no regulation of $P$. gingivalis capsule expression has been described, we can not exclude the possibility that in the in vivo situation capsule expression is regulated. However, the reduced pro-inflammatory host's immune response by the encapsulated strain may explain the documented differences between natural $P$. gingivalis strains in spreading. Whereas non-encapsulated strains are tackled directly by the immune system in localized abscesses, the more virulent encapsulated strains can evade this defence and cause phlegmonous infections [4-7].

\section{Conclusions}

The epimerase-coding gene eps $C$ of $P$. gingivalis is essential for CPS synthesis. The absence of CPS results in increased induction of $I L-1 \beta, I L-6$ and $I L-8$ in human gingival fibroblasts upon in vitro infection with viable $P$. gingivalis cells. P. gingivalis CPS acts as a functional interface between the pathogen and the host. The CPSrelated reduced pro-inflammatory response can explain why natural non-encapsulated strains cause localized abscesses and encapsulated strains spreading phlegmonous infections.

\section{Methods}

\section{Bacterial maintenance}

$P$. gingivalis strains were grown either on $5 \%$ horse blood agar plates (Oxoid no. 2, Basingstoke, UK) supplemented with hemin $(5 \mu \mathrm{g} / \mathrm{ml})$ and menadione $(1 \mu \mathrm{g} / \mathrm{ml})$ $\left(\mathrm{BA}+\mathrm{H} / \mathrm{M}\right.$ plates) or $\mathrm{BHI}+\mathrm{H} / \mathrm{M}$, both, at $37^{\circ} \mathrm{C}$ in an anaerobic atmosphere of $80 \% \mathrm{~N}_{2}, 10 \% \mathrm{H}_{2}$, and $10 \%$ $\mathrm{CO}_{2}$. Mutants were selected in the presence of $5 \mu \mathrm{g} / \mathrm{ml}$ erythromycin. Complemented mutants were selected in the presence of $50 \mu \mathrm{g} / \mathrm{ml}$ gentamycin and $1 \mu \mathrm{g} / \mathrm{ml}$ tetracycline. Purity of $P$. gingivalis liquid and plate-grown cultures was routinely checked by gram staining and microscopic examination.

Escherichia coli DH5 $\alpha$ was used for maintenance and construction of plasmids. DH5 $\alpha$ was cultured in LuriaBertani (LB) broth or on solid medium (LB broth with addition of $1.5 \%$ agar $)$. Ampicillin $\left(\mathrm{Na}^{+}\right.$salt; $\left.100 \mu \mathrm{g} / \mathrm{ml}\right)$ was added to the growth media to select for pUCderived plasmids. E. coli S17-1 grown on LB supplemented with $5 \mu \mathrm{g} / \mathrm{ml}$ tetracycline carrying the complementation construct pT-PG0120 was used for conjugation with $P$. gingivalis.

\section{Human gingival fibroblasts}

The gingival fibroblasts (HGF1 and HGF2) used in this study were collected from extracted third molars of two periodontally healthy subjects with a high pro-inflammatory immunological response when challenged with $P$. gingivalis [20]. Donors had given written informed consent, and the study was approved by the VUmc Medical Ethical committee.

\section{Genomic DNA isolation from $P$. gingivalis}

Genomic DNA from $P$. gingivalis strains was isolated from plate-grown bacteria using the DNeasy tissue kit (Qiagen Benelux BV). The DNA concentration of all samples after purification was between $20 \mathrm{ng} / \mu \mathrm{l}$ and 60 $\mathrm{ng} / \mu \mathrm{l}$.

\section{Generation of an insertional knockout construct for epsC}

To make an insertional knockout of epsC in the W83 wild type strain we constructed plasmid $\mathrm{p} \Delta \mathrm{EpsC}$. Primers epsC BamHI-F and epsC EcoRI-R (see table 1 for details) were used to amplify the $1.2 \mathrm{~Kb}$ eps $C$ gene from $P$. gingivalis W83 genomic DNA in a PCR reaction. Pfu polymerase (Fermentas, GmbH, St. Leon-Rot, Germany) was used according to the manufacturer's protocol with $100 \mathrm{ng}$ of genomic DNA. The PCR program started with $95^{\circ} \mathrm{C}$ for $5 \mathrm{~min}$ and then 25 cycles of $95^{\circ} \mathrm{C}, 55^{\circ} \mathrm{C}$ and $72^{\circ} \mathrm{C}$ for $30 \mathrm{~s}, 30 \mathrm{~s}$ and $2.5 \mathrm{~min}$ respectively and was ended by one step of $72^{\circ} \mathrm{C}$ for $5 \mathrm{~min}$. The amplified fragment was cleaned using the Qiagen PCR purification kit (Qiagen Benelux B.V.) and restricted with BamHI and EcoRI. This restricted epsC gene fragment was ligated into BamHI-EcoRI restricted pGEX-6p-3 plasmid to yield pGEX-PG0120.

The 1.2 Kb EryF erythromycin resistance cassettes for use in $P$. gingivalis was amplified from plasmid pEP4351 using primers EryF ClaI F and EryF ClaI R. and after restriction with $\mathrm{ClaI}$ this fragment was ligated into the ClaI-restricted pGEX-PG0120 plasmid yielding $\mathrm{p} \Delta \mathrm{EpsC}$. The ScaI-linearized $\mathrm{p} \Delta \mathrm{EpsC}$ plasmid was used for insertional inactivation of epsC in $P$. gingivalis strain W83.

\section{Complementation of the epsC mutant}

The 120 bp artificial constitutive CP25 promoter [37] was amplified from plasmid pDM15 [38] using primers CP25 ClaI F and CP25 AscI R. The intact epsC $1.2 \mathrm{~Kb}$ gene was amplified from genomic DNA of $P$. gingivalis strain W83 using primers epsC AscI F and epsC SpeI R. After ligation of these fragments into cloning vector pJET1.2 (Fermentas, GmbH, St. Leon-Rot, Germany) the constructed expression cassette was cut out with XhoI and HindIII and ligated into the SalI and HindIII digested pT-COW shuttle plasmid [39] to yield the complementation construct pT-PG0120. 


\section{Transformation of $P$. gingivalis}

$\mathrm{BHI}+\mathrm{H} / \mathrm{M}$ was inoculated with $P$. gingivalis W83 from a 6-day-old blood agar plate. This pre-culture was anaerobically incubated at $37^{\circ} \mathrm{C}$ for 2 days. $2 \mathrm{ml}$ of the pre-culture was used to inoculate a $100 \mathrm{ml}$ culture. The next day this culture was used to inoculate $2 \times 100 \mathrm{ml}$ of fresh $\mathrm{BHI}+\mathrm{H} / \mathrm{M}$ to an $\mathrm{OD}_{690}$ of 0.2 . After six hours of anaerobic incubation at $37^{\circ} \mathrm{C}$ the cells were harvested by centrifugation in mid-exponential phase. The pellet was washed two times in $20 \mathrm{ml}$ EPB (10\% glycerol, $1 \mathrm{mM}$ $\mathrm{MgCl}_{2}$ ) and after that resuspended in $2 \mathrm{ml}$ of EPB. Aliquots of $200 \mu \mathrm{l}$ were stored at $-80^{\circ} \mathrm{C}$ and used for electroporation.

$200 \mathrm{ng}$ of PstI digested $\mathrm{p} \Delta \mathrm{EpsC}$ was added to $200 \mu \mathrm{l}$ of W83 P. gingivalis cells. The mixture was transferred to a $2 \mathrm{~mm}$ electroporation cuvette and electroporated using an Electro Cell Manipulator 600 (BTX Instrument Division, Holliston, MA, USA; $25 \mu \mathrm{F}, 2.5 \mathrm{kV}, 186 \Omega$ ). 1 $\mathrm{ml}$ of $\mathrm{BHI}+\mathrm{H} / \mathrm{M}$ was added immediately after the pulse. The cells were left for recovery anaerobically at $37^{\circ} \mathrm{C}$ for 18 hours. The suspension was plated on $\mathrm{BA}+\mathrm{H} / \mathrm{M}$ plates with $5 \mu \mathrm{g} / \mathrm{ml}$ erythromycin for selection of the transformants. The authenticity of the insertional knockout eps $C$ mutants was verified using primer combinations epsC BamHI F $\times$ PG0119 R and EryF ClaI F $\times$ epsC EcoRI R. Furthermore, using Real-Time PCR, the expression of the downstream gene hup-1 in both W83 and the epsC mutant was monitored using primers hup$1 \mathrm{~F}$ and hup-1 $\mathrm{R}$ to exclude polar effects. W83 and the eps $C$ mutant were grown till early exponential phase. The cell pellets were collected by centrifugation and resuspended in RLT buffer (Qiagen, Benelux B. V.). The cells were disrupted using a Fast Prep Cell Disrupter (Bio 101, Thermo electron corporation, Milford, USA) and centrifuged, the total RNA was extracted from the supernatant according to the manufacturer's protocol of Qiagen $\mathrm{RNeasy}^{\circ}$ mini kit (Qiagen Benelux B.V.). The residual contaminating genomic DNA was removed by Turbo DNA-free ${ }^{\mathrm{TM}}$ kit (Ambion, Austin, USA). mRNA was then reverse transcribed using the Fermentas firststrand cDNA synthesis kit (Fermentas GmbH, St. LeonRot, Germany) according to the manufacturer's protocol.

The synthesized cDNA was further analyzed using Real-Time PCR with gene-specific primers on an ABI Prism 7000 Sequence Detecting System (Applied Biosystems, Nieuwerkerk a/d lJssel, The Netherlands). Gene expression was normalized to the expression of glucokinase ( $g l k)$, amplified with primers glk F and glk R [40]. The relative hup-1 expression levels of W83 from three independent experiments were compared in duplicate to those of the epsC mutant.

\section{Conjugation of $P$. gingivalis}

To complement the epsC mutant, plasmid pT-PG0120 was transferred into the mutant by conjugation following a protocol described earlier [41], with slight modifications. For selection of $P$. gingivalis after the over-night conjugation we used $50 \mu \mathrm{g} / \mathrm{ml}$ of gentamycin in our blood agar plates instead of $150 \mu \mathrm{g} / \mathrm{ml}$. Integrity of the trans-conjugants was confirmed by colony PCR and plasmid isolation combined with restriction analysis using a plasmid isolation kit (Qiagen Benelux B.V.).

\section{Percoll density gradient centrifugation}

Percoll density gradients were in principle prepared as described by Patrick and Reid [24]. In short, a 9:1 stock solution of Percoll (Pharmacia, Biotech AB, Uppsala, Sweden) was prepared with $1.5 \mathrm{M} \mathrm{NaCl}$. Solutions containing 80, 70, 60, 50, 40, 30, 20 and 10\% Percoll in 0.15 $\mathrm{NaCl}$ were prepared from the stock. In an open top 14 $\mathrm{ml}$ polycarbonate tube (Kontron instruments, Milan, Italy) $1.5 \mathrm{ml}$ of each of the solutions was carefully layered on top of the previous starting with $80 \% .1 \mathrm{ml}$ of an anaerobically grown over night culture of wild type and the epsC mutant concentrated to an $\mathrm{OD}_{690}$ of 4 in PBS was added to the top of the $10 \%$ layer and centrifuged for one hour at $8000 \times \mathrm{g}$ at $20^{\circ} \mathrm{C}$ in a Centrikon TST 41.14 rotor (Kontron instruments, Milan, Italy) using a Centrikon T-1170 (Kontron instruments, Milan, Italy) centrifuge.

\section{Hydrophobicty of $P$. gingivalis}

W83, the eps $C$ mutant and the complemented mutant were grown 18 hours in $\mathrm{BHI}+\mathrm{H} / \mathrm{M}$. The bacteria were washed twice in PBS after which the $\mathrm{OD}_{600}$ was set to 0.5 . After addition of $150 \mu \mathrm{ln}$-hexadecane to $3 \mathrm{ml}$ of this suspension the mix was vortexed 30 seconds, rested for 5 seconds and vortexed for 25 seconds. After exactly 10 minutes incubation at room temperature a sample was taken to measure the $\mathrm{OD}_{600}$ of the aqueous phase. The percentage of bacteria adhered to hexadecane was calculated by the formula: $\left(\mathrm{OD}_{600}\right.$ before-OD 600 after $) /$ $\mathrm{OD}_{600}$ before $\times 100 \%$. The presented data in Additional figure 1 were collected from two experiments using triplicate measurements.

\section{$P$. gingivalis serotyping}

Serotyping of $P$. gingivalis was based on the detection of the six described K-antigens [8,9]. In short, serotypespecific, polyclonal antisera were obtained after immunization of rabbits with whole bacterial cells of the six $P$. gingivalis type strains [42]. Bacterial antigens for double immunodiffusion tests were prepared as described previously [8]. Immunodiffusion was carried out in $1 \%$ agarose (Sigma Chemical Co., St. Louis, MO, type 1, low EEO) in $50 \mathrm{mM}$ Tris- $\mathrm{HCl}$ buffer $(\mathrm{pH} \mathrm{8.6).} 10 \mu \mathrm{l}$ antiserum and $10 \mu \mathrm{l}$ of antigen were loaded and allowed to diffuse and precipitate for 48 hours at room temperature.

India ink negative staining

$P$. gingivalis cells were taken from 4 day-old plates and resuspended in $1 \mathrm{ml}$ of PBS. On a glass slide $10 \mu \mathrm{l}$ of 
this suspension was mixed with $10 \mu \mathrm{l}$ of India ink (Talens, Apeldoorn, The Netherlands) and using another glass slide a thin film was made. The film was air-dried. A drop of $0.2 \%$ fuchsine was carefully added onto the film and removed after 2 minutes by decanting. Then the film was air-dried. Pictures were taken with a Leica DC500 camera on a Zeiss Axioskop using phasecontrast.

\section{Growth curve}

Pre-cultures of W83 and the epsC mutant were grown anaerobically for 18 hours in $\mathrm{BHI}+\mathrm{H} / \mathrm{M}$ at $37^{\circ} \mathrm{C}$. The pre-cultures were diluted to an $\mathrm{OD}_{690}$ of 0.05 in duplo in fresh $\mathrm{BHI}+\mathrm{H} / \mathrm{M}$ and incubated anaerobically at $37^{\circ} \mathrm{C}$. Every few hours the $\mathrm{OD}_{690}$ was measured and a sample was taken for cfu-counts.

\section{Sedimentation of $P$. gingivalis}

W83 and the epsC mutant were grown anaerobically for 18 hours in $\mathrm{BHI}+\mathrm{H} / \mathrm{M}$ at $37^{\circ} \mathrm{C}$. After 3 wash steps in phosphate buffered saline $(\mathrm{PBS})$ the $\mathrm{OD}_{690}$ was standardized to 5 in DMEM with $10 \%$ FCS. $10 \mathrm{ml}$ of this culture was added to $40 \mathrm{ml}$ DMEM with $10 \%$ FCS in a 100 $\mathrm{ml}$ flask to set the $\mathrm{OD}_{690}$ to 1 . The cultures were incubated standing still at $37^{\circ} \mathrm{C}$ for six hours. At regular time intervals, a $200 \mu \mathrm{l}$ sample was taken $0.5 \mathrm{~cm}$ from the liquid surface and the decrease of the $\mathrm{OD}_{690}$ values was determined as a measure for sedimentation.

\section{Survival of $P$. gingivalis}

W83, the epsC mutant and the complemented mutant were grown anaerobically for 18 hours in $\mathrm{BHI}+\mathrm{H} / \mathrm{M}$ at $37^{\circ} \mathrm{C}$. After 2 wash steps in phosphate buffered saline (PBS) the pellets were resuspended in DMEM with $10 \%$ FCS to an $\mathrm{OD}_{690}$ of 0.05 as used in fibroblast infections at MOI 10.000:1. $500 \mu \mathrm{l}$ of these suspensions was incubated at $37^{\circ} \mathrm{C}$ in a humidified atmosphere of $5 \% \mathrm{CO}_{2}$ in air. Samples for cfu-counts were taken at $\mathrm{t}=0$ hours, $\mathrm{t}$ $=3$ hours and $t=6$ hours and dilutions were plated on $\mathrm{BA}+\mathrm{H} / \mathrm{M}$ plates.

\section{Infection of gingival fibroblasts with $P$. gingivalis}

Bacteria were grown overnight for 18 hours in $\mathrm{BHI}+\mathrm{H} /$ M. The bacterial cells were washed three times in PBS and then used to infect gingival fibroblasts at MOIs of 1000:1 and 10.000:1 (bacteria cells: fibroblasts) in a total volume of $500 \mu \mathrm{l}$ DMEM with $10 \%$ FCS in 24-well plates. The plates were incubated for 6 hours at $37^{\circ} \mathrm{C}$ in a humidified atmosphere of $5 \% \mathrm{CO}_{2}$ in air. The cells were washed twice with cold PBS. Then $350 \mu \mathrm{l}$ lysis buffer (1\% $\beta$-mercapthanol in RLT buffer) was added to the cells according to the protocol of Qiagen RNeasy ${ }^{\oplus}$ mini kit (Qiagen Benelux B.V.) after which the plate was stored at $-80^{\circ} \mathrm{C}$ for later use.

\section{RNA isolation and reverse transcription}

mRNA was isolated from the gingival fibroblast lysates according to the manufacturer's protocol of Qiagen RNeasy $^{\oplus}$ mini kit (Qiagen Benelux B.V.). The mRNA concentrations of the samples were determined using the Nanodrop ND_1000 (Isogen Life Science). mRNA was reverse transcribed using the Fermentas first-strand cDNA synthesis kit (Fermentas GmbH, St. Leon-Rot, Germany) according to the manufacturer's protocol.

\section{Real-Time PCR}

cDNA synthesized from mRNA isolated from gingival fibroblasts after infection with $P$. gingivalis was analyzed in quadruple using Real-Time PCR with gene-specific primers on a ABI Prism 7000 Sequence Detecting System (Applied Biosystems, Nieuwerkerk a/d lJssel, The Netherlands). Reactions were performed with $2 \mathrm{ng}$ cDNA in a total volume of $8 \mu$ l containing SYBR Green PCR Master Mix (Applied Biosystems) and 0.99 pM of each primer. After activation of the AmpliTaq Gold DNA polymerase for 10 minutes at $94^{\circ} \mathrm{C}, 40$ cycles were run of a two step PCR consisting of a denaturation step at $95^{\circ} \mathrm{C}$ for 30 seconds and annealing and extension step at $60^{\circ} \mathrm{C}$ for 1 minute. Predicted product sizes were in the 100-200 bp range. Subsequently the PCR products were subjected to melting curve analysis to test if any unspecific PCR products were generated. The PCR reactions of the different amplicons had equal efficiencies. Samples were normalized for the expression of housekeeping gene $G A P D H$, which is not affected by the experimental conditions, by calculating the $\Delta \mathrm{Ct}(\mathrm{Ct}$ housekeeping gene $-\mathrm{Ct}$ gene of interest) and expression of the different genes is expressed as $2^{-(\Delta C t)}$. Fold increase in gene expression (induction) was expressed by $2^{-(\Delta \Delta \mathrm{Ct})}$, wherein $\Delta \Delta \mathrm{Ct}=\Delta \mathrm{Ct}_{\text {challenged }}$ - average $\mathrm{Ct}$-value nonchallenged.

\section{Statistical analysis}

Differences in gene induction between multiple groups were tested by one-way analysis of variance (ANOVA) and Bonferroni's Multiple Comparison Test. Tests were performed with GraphPad Prism version 4.00 for Windows, GraphPad Software, San Diego California USA. Differences were considered significant at $\mathrm{p}<0.01$.

\footnotetext{
Additional file 1: Hydrophobicity of P. gingivalis strains. Percentage of bacterial cells adhered to hexadecane after extensive vortexing and 10 minutes incubation. 3.4\%, $61 \%$ and $19 \%$ of the cells was adhered to hexadecane for W83, the epsC mutant and the complemented mutant respectively, indicating increased hydrophobicity for the epsC mutant. The data are the averages of two experiments comprised of triplicate measurements. The bars show the standard deviations.

Click here for file

[ http://www.biomedcentral.com/content/supplementary/1471-2180-10-5S1.PNG]
} 
Additional file 2: Effect of complementation of the epsC mutant on the immune response mutant of human gingival fibroblasts (HGF2) After a 6-hour challenge with $P$. gingivalis cells at $\mathrm{MOI} 10.000: 1$, the expression levels of $I L-1 \beta, I L-6$ and $I L-8$ in human gingival fibroblasts were measured using RT-PCR and if possible represented as a relative value compared to a non-infected control sample which is set to a value of 1 . Relative $I L-1 \beta$ expression could not be calculated as $I L-1 \beta$ was not detected in the non-infected control. Complementation almost restored the wild-type situation for IL-1 $\beta$ (83\%), IL-6 (83\%) and IL-8 (77\%).

Click here for file

[http://www.biomedcentral.com/content/supplementary/1471-2180-10-5S2.PNG ]

Additional file 3: Six hour survival of W83, the epsC mutant and the complemented mutant under aerobic experimental conditions.

Survival of W83, the epsC mutant and the complemented mutant in 0.5 $\mathrm{ml} \mathrm{DMEM} \mathrm{+} \mathrm{10 \%} \mathrm{FCS} \mathrm{under} \mathrm{humidified} \mathrm{5 \%} \mathrm{CO2} \mathrm{conditions} \mathrm{was}$ determined by cfu-counts on BA $+\mathrm{H} / \mathrm{M}$ plates. Survival of $67 \%, 60$ and $73 \%$ was found for each strain respectively. Error bars represent the standard deviations of triplicate measurements.

Click here for file

[http://www.biomedcentral.com/content/supplementary/1471-2180-10-5S3.PNG]

\section{Acknowledgements}

We would like to thank Jeffrey Kroon for his excellent work on the transcriptional analysis of the $P$. gingivalis genes.

\section{Author details}

'Department of Oral Microbiology, Academic Centre for Dentistry Amsterdam, Universiteit van Amsterdam and Vrije Universiteit, Amsterdam, The Netherlands. ${ }^{2}$ Center for Dentistry and Oral Hygiene, Department of Oral and Medical Microbiology, University Medical Center Groningen, Groningen, The Netherlands.

\section{Authors' contributions}

JB performed the cloning work, mutant construction, hydrophobicity test, density gradient centrifugation, negative staining, serotyping and drafted the manuscript. NBEl made the growth curves and did the sedimentation assay. NS and NBEl together performed the fibroblast infection experiments, the transcription analyses and statistical analyses. DMD analyzed the strains using Real-Time PCR and performed part of the statistical analysis. ML, AJvW and WC were involved in the study design, supervision and helped to draft the manuscript. All authors read and approved the final manuscript.

Received: 23 July 2009

Accepted: 11 January 2010 Published: 11 January 2010

\section{References}

1. Lafaurie Gl, Contreras A, Baron A, Botero J, Mayorga-Fayad I, Jaramillo A, Giraldo A, Gonzalez F, Mantilla S, Botero A, et al: Demographic, clinical, and microbial aspects of chronic and aggressive periodontitis in Colombia: a multicenter study. J Periodontol 2007, 78(4):629-639.

2. Haffajee AD, Socransky SS: Microbial etiological agents of destructive periodontal diseases. Periodontol 2000 1994, 5:78-111.

3. Page RC, Offenbacher S, Schroeder HE, Seymour GJ, Kornman KS: Advances in the pathogenesis of periodontitis: summary of developments, clinical implications and future directions. Periodontol 2000 1997, 14:216-248.

4. Grenier D, Mayrand D: Selected characteristics of pathogenic and nonpathogenic strains of Bacteroides gingivalis. J Clin Microbiol 1987, 25(4):738-740.

5. Laine ML, Appelmelk BJ, van Winkelhoff AJ: Prevalence and distribution of six capsular serotypes of Porphyromonas gingivalis in periodontitis patients. J Dent Res 1997, 76(12):1840-1844.

6. Neiders ME, Chen PB, Suido H, Reynolds HS, Zambon JJ, Shlossman M, Genco RJ: Heterogeneity of virulence among strains of Bacteroides gingivalis. J Periodontal Res 1989, 24(3):192-198.
7. van Steenbergen TJ, Delemarre FG, Namavar F, de Graaff J: Differences in virulence within the species Bacteroides gingivalis. Antonie Van Leeuwenhoek 1987, 53(4):233-244.

8. Laine ML, Appelmelk BJ, van Winkelhoff AJ: Novel polysaccharide capsular serotypes in Porphyromonas gingivalis. J Periodontal Res 1996, 31(4):278284.

9. van Winkelhoff AJ, Appelmelk BJ, Kippuw N, de Graaff J: K-antigens in Porphyromonas gingivalis are associated with virulence. Oral Microbiol Immunol 1993, 8(5):259-265.

10. Laine ML, van Winkelhoff AJ: Virulence of six capsular serotypes of Porphyromonas gingivalis in a mouse model. Oral Microbiol Immunol 1998, 13(5):322-325

11. d'Empaire G, Baer MT, Gibson FC: K1 serotype capsular polysaccharide of Porphyromonas gingivalis elicits chemokine production from murine macrophages that facilitates cell migration. Infect Immun 2006, 74(11):6236-43.

12. Farquharson SI, Germaine GR, Gray GR: Isolation and characterization of the cell-surface polysaccharides of Porphyromonas gingivalis ATCC 53978. Oral Microbiol Immunol 2000, 15(3):151-157.

13. Davey ME, Duncan MJ: Enhanced biofilm formation and loss of capsule synthesis: deletion of a putative glycosyltransferase in Porphyromonas gingivalis. J Bacteriol 2006, 188(15):5510-5523.

14. Rosen G, Sela MN: Coaggregation of Porphyromonas gingivalis and Fusobacterium nucleatum PK 1594 is mediated by capsular polysaccharide and lipopolysaccharide. FEMS Microbiol Lett 2006, 256(2):304-310.

15. Domenico P, Salo RJ, Cross AS, Cunha BA: Polysaccharide capsulemediated resistance to opsonophagocytosis in Klebsiella pneumoniae. Infect Immun 1994, 62(10):4495-4499.

16. Noel GJ, Hoiseth SK, Edelson PJ: Type b capsule inhibits ingestion of Haemophilus influenzae by murine macrophages: studies with isogenic encapsulated and unencapsulated strains. The Journal of infectious diseases 1992, 166(1):178-182

17. Glynn AA, Howard CJ: The sensitivity to complement of strains of Escherichia coli related to their K antigens. Immunology 1970, 18(3):331346

18. Aduse-Opoku J, Slaney JM, Hashim A, Gallagher A, Gallagher RP, Rangarajan M, Boutaga K, Laine ML, van Winkelhoff AJ, Curtis MA: Identification and characterization of the capsular polysaccharide (Kantigen) locus of Porphyromonas gingivalis. Infect Immun 2006, 74(1):449460.

19. Chen T, Hosogi Y, Nishikawa K, Abbey K, Fleischmann RD, Walling J, Duncan MJ: Comparative whole-genome analysis of virulent and avirulent strains of Porphyromonas gingivalis. J Bacteriol 2004, 186(16):5473-5479.

20. Scheres N, Laine ML, de Vries TJ, Everts V, van Winkelhoff AJ: Gingival and periodontal ligament fibroblasts differ in their inflammatory response to viable Porphyromonas gingivalis. J Periodontal Res 2009.

21. Schroeder HE, Munzel-Pedrazzoli S, Page R: Correlated morphometric and biochemical analysis of gingival tissue in early chronic gingivitis in man. Archives of oral biology 1973, 18(7):899-923.

22. Lekic PC, Pender N, McCulloch CA: Is fibroblast heterogeneity relevant to the health, diseases, and treatments of periodontal tissues?. Crit Rev Oral Biol Med 1997, 8(3):253-268.

23. Nagasawa $T$, Kobayashi $H$, Kiji M, Aramaki M, Mahanonda R, Kojima T, Murakami Y, Saito M, Morotome Y, Ishikawa I: LPS-stimulated human gingival fibroblasts inhibit the differentiation of monocytes into osteoclasts through the production of osteoprotegerin. Clinical and experimental immunology 2002, 130(2):338-344.

24. Patrick S, Reid JH: Separation of capsulate and non-capsulate Bacteroides fragilis on a discontinuous density gradient. J Med Microbiol 1983, 16:239241.

25. Raffatellu M, Santos RL, Chessa D, Wilson RP, Winter SE, Rossetti CA, Lawhon SD, Chu H, Lau T, Bevins CL, et al: The capsule encoding the viaB locus reduces interleukin-17 expression and mucosal innate responses in the bovine intestinal mucosa during infection with Salmonella enterica serotype Typhi. Infect Immun 2007, 75(9):4342-4350.

26. Schembri MA, Dalsgaard D, Klemm P: Capsule shields the function of short bacterial adhesins. J Bacteriol 2004, 186(5):1249-1257.

27. Shifrin Y, Peleg A, llan O, Nadler C, Kobi S, Baruch K, Yerushalmi G, Berdichevsky T, Altuvia S, Elgrably-Weiss M, et al: Transient shielding of 
intimin and the type III secretion system of enterohemorrhagic and enteropathogenic Escherichia coli by a group 4 capsule. J Bacterio/ 2008, 190(14):5063-5074

28. Dubail I, Bigot A, Lazarevic V, Soldo B, Euphrasie D, Dupuis M, Charbit A: Identification of an essential gene of Listeria monocytogenes involved in teichoic acid biogenesis. J Bacteriol 2006, 188(18):6580-6591.

29. Yoshida K, Matsumoto T, Tateda K, Uchida K, Tsujimoto S, Yamaguchi K: Induction of interleukin-10 and down-regulation of cytokine production by Klebsiella pneumoniae capsule in mice with pulmonary infection. Journal of medical microbiology 2001, 50(5):456-461.

30. Gibson FC, Tzianabos AO, Onderdonk AB: The capsular polysaccharide complex of Bacteroides fragilis induces cytokine production from human and murine phagocytic cells. Infect Immun 1996, 64(3):1065-1069.

31. Raffatellu M, Chessa D, Wilson RP, Dusold R, Rubino S, Baumler AJ: The Vi capsular antigen of Salmonella enterica serotype Typhi reduces Toll-like receptor-dependent interleukin-8 expression in the intestinal mucosa. Infect Immun 2005, 73(6):3367-3374.

32. Vann WF, Daines DA, Murkin AS, Tanner ME, Chaffin DO, Rubens CE, Vionnet J, Silver RP: The NeuC protein of Escherichia coli K1 is a UDP Nacetylglucosamine 2-epimerase. J Bacteriol 2004, 186(3):706-712.

33. Wilson RP, Raffatellu M, Chessa D, Winter SE, Tukel C, Baumler AJ: The Vicapsule prevents Toll-like receptor 4 recognition of Salmonella. Cellular microbiology 2008, 10(4):876-890.

34. Sojar HT, Hamada N, Genco RJ: Isolation and characterization of fimbriae from a sparsely fimbriated strain of Porphyromonas gingivalis. Applied and environmental microbiology 1997, 63(6):2318-2323.

35. Krinos CM, Coyne MJ, Weinacht KG, Tzianabos AO, Kasper DL, Comstock LE: Extensive surface diversity of a commensal microorganism by multiple DNA inversions. Nature 2001, 414(6863):555-558.

36. Tran QT, Gomez G, Khare S, Lawhon SD, Raffatellu M, Baumler AJ, Ajithdoss D, Dhavala S, Adams LG: The Salmonella enterica serotype Typhi Vi capsular antigen is expressed after entering the ileal mucosa. Infect Immun 2010, 78(1):527-35.

37. Jensen PR, Hammer K: The sequence of spacers between the consensus sequences modulates the strength of prokaryotic promoters. Applied and environmental microbiology 1998, 64(1):82-87.

38. Deng DM, Liu MJ, ten Cate JM, Crielaard W: The VicRK system of Streptococcus mutans responds to oxidative stress. J Dent Res 2007, 86(7):606-610.

39. Gardner RG, Russell JB, Wilson DB, Wang GR, Shoemaker NB: Use of a modified Bacteroides -Prevotella shuttle vector to transfer a reconstructed beta-1,4-D-endoglucanase gene into Bacteroides uniformis and Prevotella ruminicola B(1)4. Applied and environmental microbiology 1996, 62(1):196-202.

40. Diaz PI, Slakeski N, Reynolds EC, Morona R, Rogers AH, Kolenbrander PE: Role of oxyR in the oral anaerobe Porphyromonas gingivalis. J Bacteriol 2006, 188(7):2454-2462.

41. Belanger M, Rodrigues P, Progulske-Fox A: Genetic manipulation of Porphyromonas gingivalis. Current protocols in microbiology 2007, Chapter 13(Unit13C):12.

42. van Winkelhoff AJ, Kippuw N, de Graaff J: Serological characterization of black-pigmented Bacteroides endodontalis. Infect Immun 1986, 51(3):972974.

doi:10.1186/1471-2180-10-5

Cite this article as: Brunner et al: The capsule of Porphyromonas gingivalis reduces the immune response of human gingival fibroblasts. BMC Microbiology 2010 10:5.

Publish with Bio Med Central and every
scientist can read your work free of charge

"BioMed Central will be the most significant development for disseminating the results of biomedical research in our lifetime. "

Sir Paul Nurse, Cancer Research UK

Your research papers will be:

- available free of charge to the entire biomedical community

- peer reviewed and published immediately upon acceptance

- cited in PubMed and archived on PubMed Central

- yours - you keep the copyright 